\title{
Big Data-based the Smart Grid Application Analysis
}

\author{
Zhengguang Liu ${ }^{1, ~ *}$, Qiaoyu Liu ${ }^{1}$, Mengjiang $\mathbf{W u}^{2}$ \\ ${ }^{1}$ College of Water Resources and Architectural Engineering, Northwest A \& F University, Yangling, China \\ ${ }^{2}$ Electrical Department, College of Science and Technology of China Three Gorges University, Yichang City, China
}

\section{Email address:}

zhengguangliu@nwafu.edu.cn (Zhengguang Liu)

*Corresponding author

\section{To cite this article:}

Zhengguang Liu, Qiaoyu Liu, Mengjiang Wu. Big Data-based the Smart Grid Application Analysis. International Journal of Industrial and Manufacturing Systems Engineering. Vol. 4, No. 4, 2019, pp. 41-47. doi: 10.11648/j.ijimse.20190404.12

Received: October 4, 2019; Accepted: October 21, 2019; Published: October 25, 2019

\begin{abstract}
The smart grid is the future development direction of the power industry. The ultimate goal of the smart grid should be to build a real-time monitoring system covering the entire production process of the power system, including power generation, transmission, power transmission, power distribution, and power scheduling. A smart grid is the development trend of the future power industry, and the application analysis of the smart grid is the basis for ensuring economic and safe operation. Smart Grid Application Analysis (SGAA) based on big data, is of considerable significance to the development of the power system. Based on the comprehensive comparison of domestic and foreign literature, this paper puts forward the prediction application of "Big Data + " and makes a simple evaluation of the possible potential power-side load and regulator prediction model of new energy development. It also introduces the shortcomings in the current stage, as well as the critical technologies of the big data industry that need to be developed urgently. The smart grid is the future direction of power industry development, but the current stage of the development of related technology is not enough, this paper gives suggestions for the development of smart grid and big data.
\end{abstract}

Keywords: Industrial Applications, Big Data, Smart Grids

\section{Introduction}

The smart grid is the future development direction of the power industry. The ultimate goal of the smart grid should be to build a real-time monitoring system covering the entire production process of the power system, including power generation, transmission, power transmission, power distribution, and power scheduling. Through the application of information and communication technology, computer technology, control technology, and other technologies, the coordination of the needs and functions of all stakeholders in power plants, power grids, and power markets is accomplished, which will improve the operational efficiency of various parts of the system and realize the cost reduction. The basis of the reliable operation of the smart grid lies in the rapid analysis of the massive multi-source data accumulated by the grid, of which the forecast analysis of the smart grid is essential. By applying big data technology to smart grid prediction and analysis, we can improve prediction accuracy, which is of considerable significance to the operation of power system.

Big data technology has received widespread attention in recent years, which refers to the technology system that captures, discovers and analyzes a large number of kinds and sources of complex data at high speed and extracts its value reasonably. The smart grid is regarded as one of the essential technical fields of big data applications. On the one hand, a large number of intelligent meters and broad application of sensing technology, the power industry has produced a large number of diverse structures, multiple sources of data, how to store and apply these data, is a difficult problem facing power companies. On the other hand, using these data can expand more value-added services for power companies and reduce the environmental impact.

Since 2012, many attempts have been made in the research and engineering application of big data technology in smart grid, but on the whole, these efforts are still in the early stages of exploration. Based on comparing domestic and foreign research, this paper introduces big data and smart 
grid, and puts forward the predictive concept of "Big data + ". With analyze of the possible load and steady-state voltage stability prediction of new electric energy development, the research summarizes the existing problems of data collection and puts forward the research framework and technical route of the big data of the smart grid.

\section{Literature Review}

By comparing different kinds of literature, we can learn that the development trend of the smart grid in recent years, such as in the literature $[17,22]$, the construction model of the smart grid, and give some constructive opinions. In the literature considering the critical technologies of big data such as data visualization, such as literature [2, 4], which discuss the combination of data mining, data analysis, and other astronomical data specific analysis techniques. Smart grid technology is also analyzed and predicted. Some of the literature, like [17], produces the model prediction of artificial intelligence and smart grid. This paper has an apparent reference for the combination of big data algorithms and smart grids, such as deep learning, and in the literature, through the prediction of new energy, user-side load prediction (literature [1] is an accurate load analysis of power system.)

The analysis of the forecast model of the load side and the steady-state voltage prediction is made in literature [17], and the smart grid prediction model is evaluated, which further reflects the application direction of big data in the smart grid. As for the development of related technologies, the literature [18] can draw the direction of the integration of big data technology and industry in recent years, such as in the literature [8], it reflects the combination of smart grid and smart building, and the literature [21], which further shows that the smart grid can play a decisive role in the user pricing process, and the comparison and analysis of these papers, The document [4] puts forward the structure of the information system, and the document gives the relevant development suggestions and guidance from the policy perspective and the leadership level.

These documents can help readers understand the direction of the convergence of big data technology and smart grid, promote the convergence of big data technology and smart grid, and realize the reliable and accurate big data and smart grid.

\section{Predictive Application Analyze}

By comparing the research literature of big data and smart grid, we can see that a large number of shallow models have been applied to new energy and load prediction, such as in some references, the advantages and limitations of the existing shallow models are summarized in a better manner. As mentioned earlier, shallow models have limited data analysis capacity for multiple sources of power grids and are prone to complex classification problems, while a large number of new energy access to the grid will cause uncertainty of grid data, and user behavior is complex and challenging to describe, which makes the application of relevant shallow models restricted. From the current development, the application of new energy generation is bound to usher in the application of big data, and we can use the same method in the application research, the relevant forecast model scans into new energy forecast, user load forecast and steady-state prediction. Through the analysis of these, we can quickly get the future development direction of the smart grid and analyze the potential application of big data.

\subsection{New Energy Application}

The main components of the current new energy generation are photovoltaic and wind power generation, geothermal power generation, tidal power generation, wave power generation, and so on. The common problem with these methods is that the power generation time is not fixed and the power quality is not guaranteed. The forecast of the new energy generation analyzed in this paper mainly includes the prediction of photovoltaic power and wind power, as well as the corresponding irradiance and wind speed forecast. Because of the intermittent and volatile irradiance and wind speed, the prediction model must have high nonlinear timing characteristics.

It can be seen from the reference that current research is applied in three ways, the first is to use RNN and LSTM algorithms that can process time-series data, and after comparing the prediction accuracy of photovoltaic power under different models, the model with the highest prediction accuracy is obtained. For photovoltaic prediction, LSTM application can be used to process timing PV data, more applications and better results, the second is often using other monitoring learning algorithms to process data, such as wavelet transformation and other methods, which can decompose the original PV power signal into different frequency sequences, and then for each sequence of deterministic PV prediction, Make probability PV prediction. The last method is to use the deep characteristics of unsupervised learning or extract data, and then use a supervised learning algorithm to complete the prediction function. This method tends to be computationally extensive, and the algorithm is more complex.

Through the above algorithm conclusion analysis, it can be seen that big data in photovoltaic power generation industry, wind power generation industry and other aspects of a large number of applications, and because of the industry's instability and uncontrollable, the emergence of big data will have uncontrollable and timely nature.

\subsection{Power Side Prediction}

In order to accurately obtain the user's power data, the power company deployed a large number of smart meters with two-way communication capability, which can send real-time power information to the grid every $5 \mathrm{~min}$ frequency, which provides the conditions for the implementation of household load prediction. However, in 
recent years, the disorderly charging and discharge behavior of electric vehicles has brought trouble to the efficient collection of power grid data, and if we can monitor the charging and discharge status of electric motor vehicle batteries with a broad base, the active collection of data can be achieved.

For big data that has been collected, predictive estimates can be made using the same algorithms as in the forecast of new energy generation. However, compared with the system-level load, there are outliers and discontinuities in the family-level load, so it has variability and uncertainty, which shows that LSTM with learning timing association ability is more suitable for family-level load prediction. At the same time, because the forecast of power grid load is related to social factors such as holidays and weather, more attention should be paid to the combination of data mutation and different types of data when making the electricity-side forecast.

At present, there have been many technical methods for load prediction for big data, mainly multi-linear back. The return and semi-parameters can be added model, exponential smoothing model, fuzzy regression model, gradient lift model, etc., which are mostly applied to point load prediction. However, there are still fewer spatial load prediction models.

From the current model, the user-side change is not high, but in the application of electric vehicles, most of the statistics are still incomplete, as an indispensable part of the application of big data industry, there is no doubt that the combination of smart grid and big data technology will be well applied on the user side.

\subsection{Steady-state of Power System}

At present, more research on steady-state voltage stability prediction focuses on voltage stability margin (Voltage Stability Margin, VSM) online prediction, which is generally obtained by Continues Power Flow calculation and is generally affected by the initial state operation point of the power system, the direction of load growth, etc. These predictions are widely studied and applied for real-time monitoring. Steady-state voltage prediction has always been a mature research field in the power system, but it has also been producing new methods. However, there is still much work worth further study and discussion considering various types of data, various technical algorithms and different levels of system network structure. These factors make big data technology is full of uncertainty.

Through the above prediction, it can be seen that the combination of big data technology and smart grid will establish from power generation, transmission, to power distribution, electricity, four-in-one real-time monitoring system.

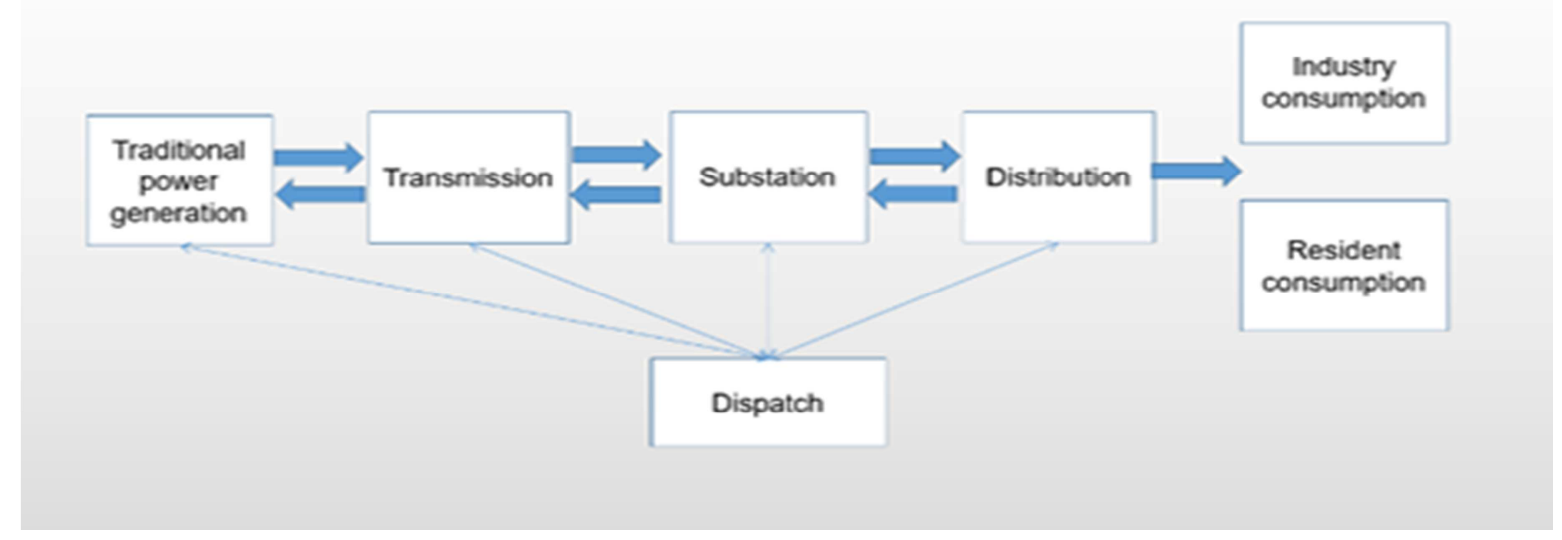

Figure 1. Traditional Grid.

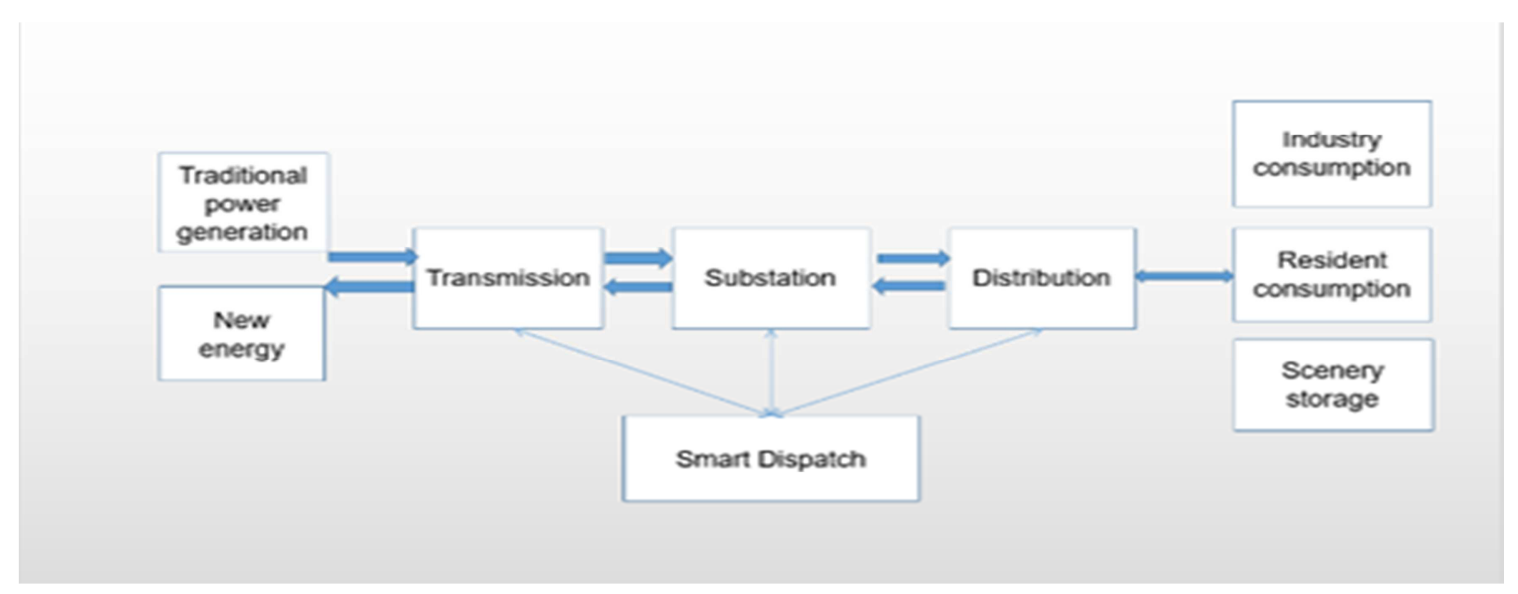

Figure 2. Smart Grid. 


\section{Key Technologies}

The key to the prediction implementation mentioned above lies in the execution of data mining, data analysis, and other processes. As a critical technology in big data technology, these technologies play an essential role in the integration of big data and smart grid. There are many shortcomings in the data analysis and processing technology of big data, which is mainly stored in underutilization, and this paper summarizes the application technology of big data in smart grid from the aspects of data storage processing and data analysis.

\subsection{Storage Platform}

From the relationship between ample data storage and processing, there are two central storage and processing modes. One is direct processing when new data arrives, it is processed immediately and returns the required results, suitable for real-time demanding businesses, can be used for power and load joint scheduling and on-line monitoring of equipment.

The other is storage and processing, which is suitable for real-time businesses that are not high in real-time but have a vast amount of data. A smart grid is an ever-evolving system engineering, which cannot guarantee its feasibility, reliability, and scalability by merely centralizing the data logically to control it.

The distributed data processing system, database, and other cloud computing technology can be used as the primary platform for massive data storage, for the application of big data in the smart grid services.

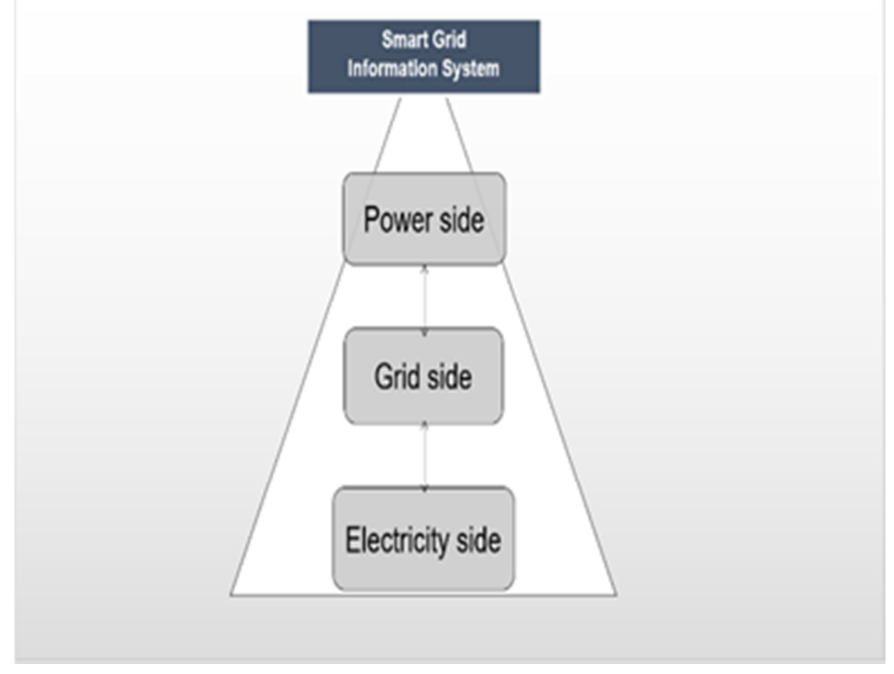

Figure 3. Smart Grid Information System.

\subsection{Data Analysis}

Data analysis, including data analysis and interpretation of two aspects. By deeply analyzing the computer big data itself and presenting itself in many dimensions, the results of the analysis can be restored to specific industry problems. The results of the analysis are further understood and applied. However, due to the limitations of computer technology, the calculation power improvement has not yet been popularized, so the process of interpretation still needs to be further improved.

Extracting useful information from large data sets or data streams generated by the grid and further integrating multiple data or knowledge describing power systems into a consistent and accurate industry issue. Through the mining and integration of data from different sources, the potential relationship between data can be further explored, such as changes in night-time electricity consumption can reflect the development of the local urban economy. More accurate conclusions are drawn through a more accurate understanding of grid data.

Although the grid generates and accumulates many data during its operation, most of which can no longer continue to create value after its utilization cycle ends; in fact, some seemingly outdated, outdated, and useless data still has potential value. For example: the long-term and large number of electric vehicle charging data generated by electric vehicle charging information is of great reference value to the charging station, the user's electricity consumption has long been mainly used as the basis of load statistics and user billing, and the extended analysis found that there is a specific relationship between this data and housing vacancy rate.

In the operation and planning process of the smart grid, there are often some experience or laws, although the generality is extreme, but has not yet or can not rise to the theoretical level, can not be displayed in a natural form in front of people. The analysis and mining of big data in smart grid can obtain universal knowledge hidden in habits or experience, and help operators and planners improve their awareness of the whole smart grid. Among them, the process mining of smart grid can not only guide the user to use the wrong peak electricity, avoid the failure of the power system, but also for the user to develop a more reasonable energy-saving plan, improve the management level of the power grid enterprises, optimize the service of the power grid.

By using computer graphics and image processing technology, the analysis conclusions of big data are converted into graphics or images and presented to the user, and by combining data visualization technology with big data analysis technology, the complete data set can be given, which can further show the development trend of grid data.

You can also selectively provide valuable information to grid business units or users; In addition, data visualization can further amplify the user's demand-driven, such as selecting the floating tariff that the user is more concerned about, according to the consumption characteristics of different residential users' power and the user's energy consumption level, thereby further optimizing the power company's services. The dynamic estimation and micro-display of the evolving change points in the power 
grid can be used for visual emergency response of extreme weather.

Data visualization, as a data analysis tool and results presentation method, can most intuitively reflect the application of the smart grid in big data and application value.

\section{Motivators and Obstacles}

Although the application of the technology mentioned above is helpful to the construction of smart computers, there are still different factors in the current industrial system, which play a role in promoting or hindering the construction of smart grid, and a reasonable analysis of such factors will help us to recognize the status quo further and promote the progress of technology.

\subsection{Development Drivers}

In 2019, China's State Grid proposed the construction of a smart and robust power grid and a generalization of the Internet of Things. Before this, countries around the world have long-established relevant laws and regulations to promote the construction of smart grid. At the same time, as early as the end of 2008, Europe has released the European Smart Grid Strategic Development Plan, and in April 2010 released the final version, the development of the European smart grid by priority divided into six levels, covering grid optimization, distributed energy, information and communication technology to market operations and so on. The United States, for its part, set up a new smart grid pilot in Maui, Hawaii, as early as May 2011. In addition to the support of relevant policies, the market demand for high-quality electricity is also rising. At the same time, due to the full application of smart meters, the user-side data has grown widely. Big Data Technology and Smart Grid Technology integration are inevitable.

However, from the general point of view, the current foreign smart grid research focuses on distributed energy access and power generation power side interaction, China's smart grid research work focuses on the massive power grid system information acquisition and stability control, which may be related to the characteristics of strong coupling of China's power network.

\subsection{Development Impediments}

The combination of smart grid and big data is a combination of grid analysis and operation technology, data mining and analysis technology and IT technology, with comprehensive and complex. Although some decentralized research and applications have been carried out in the field of big data at home and abroad, as far as the power industry is concerned, the whole power company has not yet worked out the big data strategy of the whole enterprise, and it is still in the initial stage of exploration.

At the same time, because of the common problem of interoperability in data exchange, the technical differences and standard differences between hardware and software systems have brought about interoperability problems.

Big data research and development require much investment, but the benefits are not yet clear, and researchers need to establish further the fund recovery model and evaluation methods of big data applications to stimulate the continued investment of funds.

At the same time, in order to ensure that the existing big data technology can be fully capable, the information and communication architecture needs to be further adjusted to meet the development needs of big data, and this also needs to invest in related funds.

The difficulty of obtaining data comes not only from hardware constraints but also from existing management mechanisms. At present, the data of the power industry is stored in different data formats in different databases, which is shaft-shaped, which makes it difficult to exchange and share data.

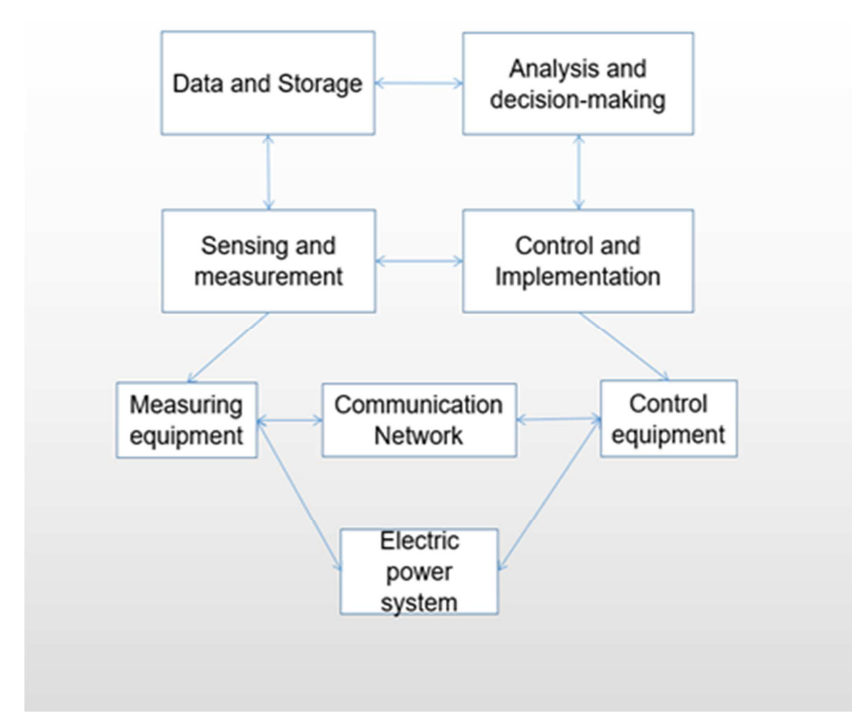

Figure 4. Supporting Platform of Smart Gird Information.

Some data power companies are not allowed to use publicly; some data need to be used to a limited extent, privacy protection and security is the big data in the construction of smart computer first problem. Power professionals are not aware of the basic concept of big data, the application value of big data in the smart grid, and need convincing results to be generally recognized.

\section{Development Proposals}

Based on the development and application of big data technology, combined with the actual development of the existing smart grid, the following suggestions are made for the development of big data in China's smart grid.

1. Improve the knowledge system, technology system and research framework of big data in smart grid, and further train the professional team engaged in big data research in smart grid technology, while continuing to carry out in-depth analysis of big data technology needs 
in the field of smart grid technology, scene design, application value analysis, and complete the research and development of the big data platform of smart grid.

2. Focus on data access and convergence technology research on big data in the smart grid, including communication information system construction, data security, and privacy protection-related technologies, smart grid power quality improvement. At the same time, based on data integration, we will research big data applications with users collecting data from electricity information as the core, which lays the foundation for more extensive technical research and accumulates experience.

3. Further develop new and renewable energy generation, and study how to further improve the data quality and data fusion of big data in the smart grid, and expand the scope of data fusion. Conduct comprehensive research into big data applications in other technology areas.

\section{Conclusion}

1. The smart grid is an important application area of big data. With the construction of a smart grid, a large number of measurement and monitoring data have been produced, how to deal with the value of mining is a problem faced by power companies.

2. Big data is a comprehensive technology, through the comparative analysis of existing research, the new energy generation, power side load, regulator prediction, and other models have been analyzed, and with the formation of new theories and methods, will further promote the revolution of the power industry. Power workers should closely track the development of big data theory and technology, in order to make effective use of big data technology, do a good job in the ideological and technological reserves.

3. Smart grid big data research and engineering applications are just beginning is still in the exploration stage; all kinds of key technologies are not yet mature. Efforts are needed to promote the healthy development of the smart grid. The research and engineering application of big data in smart grid also needs to go through a long process of development.

\section{References}

[1] Yu Xin, Lu Jianming, He Pengcheng, Cai Zijian. Research on Smart Grid Load Prediction Algorithms. J. Computer and Digital Engineering, 2019 (09): 2357-2363.

[2] Ismaila Adeniyi Kamil, Sunday Oyinlola Ogundoyin. A big data anonymous batch verification scheme with conditional privacy preservation for power injection over vehicular network and 5G smart grid slice [J]. Sustainable Energy, Grids and Networks, 2019.

[3] Ni Zhen, Paul Shuva. A Multistage Game in Smart Grid Security: A Reinforcement Learning Solution. [J]. IEEE transactions on neural networks and learning systems, 2019, 30 (9).
[4] Anees Junaid, Zhang Hao-Chun, Baig Sobia, Lougou Bachirou Guene. Energy-Efficient Multi-Disjoint Path Opportunistic Node Connection Routing Protocol in Wireless Sensor Networks for Smart Grids. [J]. Sensors (Basel, Switzerland), 2019, 19 (17).

[5] Saqib Hasan, Abhishek Dubey, Gabor Karsai, Xenofon Koutsoukos. A game-theoretic approach for power systems defense against dynamic cyber-attacks [J]. International Journal of Electrical Power and Energy Systems, 2020, 115.

[6] Li Tao, Yan Gao. Real-time pricing for smart grid with distributed energy and storage: A noncooperative game method considering spatially and temporally coupled constraints $[\mathrm{J}]$. International Journal of Electrical Power and Energy Systems, $2020,115$.

[7] Per-Anders Langendahl, Helen Roby, Stephen Potter, Matthew Cook. Smoothing peaks and troughs: Intermediary practices to promote demand side response in smart grids [J]. Energy Research \& Social Science, 2019, 58.

[8] Zhang Yanjun, Yang Xiaodong, Liu Yi, Zheng Dayuan, Bi Shujun. Research on the Frame of Intelligent Inspection Platform Based on Spatio-temporal Data. Computer \& Digital Engineering [J], 2019, 47 (03): 616-619+637.

[9] Ima Essiet, Yanxia Sun, Zenghui Wang. Scavenging differential evolution algorithm for smart grid demand side management [J]. Procedia Manufacturing, 2019, 35.

[10] Energy; Studies from Hong Kong Polytechnic University Update Current Data on Energy (Game Theory Based Interactive Demand Side Management Responding To Dynamic Pricing In Price-based Demand Response of Smart Grids) [J]. Energy Weekly News, 2019.

[11] Francesco D' Ettorre, Mattia De Rosa, Paolo Conti, Daniele Testi, Donal Finn. Mapping the energy flexibility potential of single buildings equipped with optimally-controlled heat pump, gas boilers and thermal storage [J]. Sustainable Cities and Society, 2019, 50.

[12] Yi Liu, Jiawen Peng, and Zhihao Yu. 2018. Big Data Platform Architecture under The Background of Financial Technology: In The Insurance Industry As An Example. In Proceedings of the 2018 International Conference on Big Data Engineering and Technology (BDET 2018). ACM, New York, NY, USA, 31-35.

[13] Mohammad Taheri Tehrani, Ali Mohammad Afshin Hemmatyar. Welfare-aware strategic demand control in an intelligent market-based framework: Move towards sustainable smart grid [J]. Applied Energy, 2019, 251.

[14] L. Yi and W. Yi, "Decision Tree Model in the Diagnosis of Breast Cancer," 2017 International Conference on Computer Technology, Electronics and Communication (ICCTEC), Dalian, China, 2017, pp. 176-179. doi: 10.1109/ICCTEC.2017.00046.

[15] Hamed Hashemi-Dezaki, Ali-Mohammad Hariri, Maryam A. Hejazi. Impacts of load modeling on generalized analytical reliability assessment of smart grid under various penetration levels of wind/solar/non-renewable distributed generations [J]. Sustainable Energy, Grids and Networks, 2019, 20.

[16] Amevi Acakpovi, Ruhiya Abubakar, Nana Yaw Asabere, Issah B. Majeed. Barriers and Prospects of Smart Grid Adoption in Ghana [J]. Procedia Manufacturing, 2019, 35. 
[17] Wu Weihong, Han Yu, Feng Lin, Li Guojie, Jiang Xiuchen. "AI+" based smart grids prediction analysis Journal of Shanghai Jiaotong University, 2018, 52 (10): 1206-1219.1266.

[18] Zhang Dongxia, Miao Xin, Liu Liping, Zhang Yan, Liu Scientific Research. Smart Grid Big Data Technology Development Research. J, China Motor Engineering News, 2015, 35 (01): 2-12.

[19] Song Yaqi, Zhou Guoliang, Zhu Yongli. Present Status and Challenges of Big Data Processing in Smart Grid. Grid Technology, 2013, 37 (04): 927-935.

[20] Hu Xuehao. Smart grid: a development trend of future power grid [J]. Power System Technology, 2009, 33 (14): 1-5 (in Chinese).

[21] Zhou Hui, Niu Wenjie, Wang Yi. Analysis of clients' credit based on theirs paying behaviors [J]. Power Demand-Side Management, 2006, 8 (6): 12-16 (in Chinese).

[22] Zhang Guangbin, Shu Hongchun, Yu Jilai. Travelling wave field data contingency screening based on semi-supervised clustering using generalized current modal component [J]. Proceedings of the CSEE, 2012, 32 (10): 150-158 (in Chinese).

[23] Xi Fang, Satyajayant Misra, Guoliang Xue, et al. Smart Grid, the new and improved power grid: a survey [J]. IEEE Communications Surveys and Tutorials (COMST), 2012, 14 (4): 944-980.

[24] Z. Zhao, J. Wang and Y. Liu, "User Electricity Behavior Analysis Based on K-Means Plus Clustering Algorithm," 2017 International Conference on Computer Technology, Electronics and Communication (ICCTEC), Dalian, China, 2017, pp. 484-487. doi: 10.1109/ICCTEC.2017.00111. 